\title{
Singular Elastic Properties of Mixtures with the Re-entrant Nematic Phase
}

\author{
G. Czechowski And J. JADŻYN \\ Institute of Molecular Physics, Polish Academy of Sciences \\ M. Smoluchowskiego 17, 60-179 Poznań, Poland
}

\begin{abstract}
(Received June 3, 2004)
The paper presents temperature behavior of the splay and bend elastic constants measured for $n$-hexyloxycyanobiphenyl and $n$ octyloxycyanobiphenyl mixtures - a system exhibiting the re-entrant nematic phase. It was shown that singularities in the elastic properties of the system concern not only the nematic phase (the well-known pre-smectic effects) but first of all - the smectic $A$ phase, in which the splay and bend deformations, normally not allowed for the layered structures, can be induced.
\end{abstract}

PACS numbers: 64.70.Md, 62.20.Dc, 61.30.--v

\section{Introduction}

An unusual sequence, discovered nearly thirty years ago, of the mesophases: nematic $(N)$ - smectic $A\left(S_{A}\right)$ - re-entrant nematic $\left(N_{R}\right)$, observed with decreasing temperature (or increasing pressure) of some mesogenic systems [1, 2], is still a subject of many investigations, mainly experimental [3-9]. The re-entrance of the mesophase of a higher symmetry, at temperature below an existence of a stable phase of lower symmetry, is a fascinating phenomenon the more so as the similar effects are observed for superconductors and helium [10].

Various theoretical models of the re-entrance of nematic phase on the molecular level have been proposed. Most of them is based on the existence of the monomers - dimers equilibrium in mesogenic liquids composed of strongly polar molecules (like cyanobiphenyl derivatives) [11-16]. The nematic phase re-appears when, with decreasing temperature, the concentration of dimers reaches some critical value. A more general approach takes into account a sensitive balance between dipolar and steric factors [17]. 
Visco-elastic properties of the systems in which the re-entrance of the nematic phase occurs are a subject of not too numerous papers [1,3, 9, 18-20]. The main problem of the papers, anyway it concerns not only the visco-elastic properties, was the detailed analysis of the physical properties of the two nematic phases occurring in the system. In the prevailing number of the papers it was found that the properties of the nematic and re-entrant nematic phases are convergent to each other or are just identical. In particular, in the vicinity of the nematic to smectic $A$ phase transition, temperature behavior of the elastic constants in both nematic phases shows a far-reaching similarity. Because in the most papers the light scattering method was used for measuring the elastic constants, the smectic $A$ phase situated between two nematic phases was excluded from the investigations because the external stimulus was not strong enough to induce the deformation in that layered phase.

The elastic properties of the smectic $A$ phase in the systems exhibiting the re-entrant nematic phase, i.e., showing the $N-S_{A}-N_{R}$ sequence of the phases, are the main subject of the present paper. The measurements were performed for the well-known mixtures of $n$-hexyloxycyanobiphenyl (6OCB) and $n$ octyloxycyanobiphenyl (8OCB). The elastic constants corresponding to the splay $\left(K_{11}\right)$ and bend $\left(K_{33}\right)$ deformations were determined with the use of the capacitance method, where a relatively strong external stimulus (the electric field) can be applied to the system.

\section{The capacitance method for determination of the elastic constants}

The method consists in the analysis of the voltage dependence of the capacity of the planar nematic cell. In case of nematic liquid crystals with a positive dielectric anisotropy, $\Delta \varepsilon=\varepsilon_{\|}-\varepsilon_{\perp}>0$, where $\varepsilon_{\|}$and $\varepsilon_{\perp}$ are the electric permittivities measured, respectively, parallel and perpendicular to the macroscopic molecular ordering (the director $\boldsymbol{n}$ ), the probing electric field $\boldsymbol{E}$ of a sufficient intensity causes a distortion of the director (Fig. 1). With increasing field intensity, the capacity of the cell increases from the initial value $C_{\perp}$ - corresponding to $\boldsymbol{n} \perp \boldsymbol{E}$, to the final value $C_{\|}-$corresponding to $\boldsymbol{n} \| \boldsymbol{E}$. The phenomenon has the threshold, i.e., the capacity does not change itself before the voltage reaches characteristic of a given nematic liquid crystal value $U_{\text {th }}$ (Freedericksz transition). This value is the basic quantity for calculation of the splay elastic constant:

$$
K_{11}=\frac{\varepsilon_{0} \Delta \varepsilon U_{\mathrm{th}}^{2}}{\pi^{2}},
$$

where $\varepsilon=8.85 \mathrm{pF} / \mathrm{m}$.

The elastic constant $K_{33}$ can be determined in the same experiment, from the analysis of a dependence of the cell capacitance on the voltage above the threshold value. The exact equation describing the $C(U)$ dependence was obtained first by Gruler, Scheffer and Meier [21]. Next, Uchida and Takahashi [22] proposed 


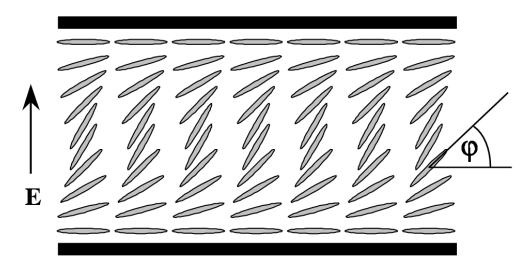

Fig. 1. Distorsion of the director $\boldsymbol{n}$ in the planar nematic cell caused by the voltage higher than the Freedericksz threshold value $U_{\text {th }}$.

a procedure for the $K_{33}$ determination, which eliminates the need of a multi-parameter least square fitting of the Gruler et al. equation to the experimental data. The final equation for $C(U)$ dependence has a following form [23, 24]:

$$
\begin{aligned}
& \frac{C(U)-C_{\perp}}{C_{\perp}}=\gamma-\frac{2 \gamma}{\pi} \frac{U_{\mathrm{th}}}{U}\left(1+\gamma \sin ^{2} \varphi_{m}\right)^{1 / 2} \\
& \quad \times \int_{0}^{\varphi_{m}}\left[\frac{\left(1+\chi \sin ^{2} \varphi\right)\left(1-\sin ^{2} \varphi\right)}{\left(1+\gamma \sin ^{2} \varphi\right)\left(\sin ^{2} \varphi_{m}-\sin ^{2} \varphi\right)}\right]^{1 / 2} \cos \varphi \mathrm{d} \varphi
\end{aligned}
$$

where $\chi=K_{33} / K_{11}-1, \gamma=\varepsilon_{\|} / \varepsilon_{\perp}-1, \varphi$ is the tilt angle between the director $\boldsymbol{n}$ and the cell walls (see Fig. 1) and $\varphi_{m}$ is the tilt at the center of the cell.

For the voltage much higher than the threshold value, the director at the center of the cell becomes perpendicular to the cell walls and $\varphi_{m}=\pi / 2$. Then, Eq. (2) reduces to

$$
\frac{C(U)-C_{\perp}}{C_{\perp}}=\gamma-\frac{2 \gamma}{\pi} \frac{U_{\mathrm{th}}}{U}(1+\gamma)^{1 / 2} \int_{0}^{\pi / 2}\left(\frac{1+\chi \sin ^{2} \varphi}{1+\gamma \sin ^{2} \varphi}\right)^{1 / 2} \cos \varphi \mathrm{d} \varphi .
$$

This equation predicts that for $U \gg U_{\text {th }}$ the dependence $\left(C-C_{\perp}\right) / C_{\perp}$ on $U^{-1}$ should be linear. The extrapolation of the dependence to $U^{-1}=0$ leads directly to the value of $\gamma=\Delta \varepsilon / \varepsilon_{\perp}$ and the slope

$$
\alpha=\frac{2 \gamma}{\pi}(1+\gamma)^{1 / 2} U_{\mathrm{th}} \int_{0}^{\pi / 2}\left(\frac{1+\chi \sin ^{2} \varphi}{1+\gamma \sin ^{2} \varphi}\right)^{1 / 2} \cos \varphi \mathrm{d} \varphi
$$

contains only one unknown quantity $\chi=K_{33} / K_{11}-1$. Hence, the $K_{33}$ elastic constant can be easily obtained since the $K_{11}$ constant is known from Eq. (1).

\section{Experimental}

$n$-Hexyloxycyanobiphenyl $\left(\mathrm{C}_{6} \mathrm{H}_{13} \mathrm{O}-\mathrm{PhPh}-\mathrm{C} \equiv \mathrm{N}, 6 \mathrm{OCB}\right)$ with the following sequence of the phase transitions: crystal $(\mathrm{Cr})-58^{\circ} \mathrm{C}-\operatorname{nematic}(N)-76^{\circ} \mathrm{C}$ - isotropic $(I)$ and $n$-octyloxycyjanobiphenyl $\left(\mathrm{C}_{8} \mathrm{H}_{17} \mathrm{O}-\mathrm{PhPh}-\mathrm{C} \equiv \mathrm{N}, 8 \mathrm{OCB}\right)$ : $(C r)-54.5^{\circ} \mathrm{C}-$ smectic $A\left(S_{A}\right)-66^{\circ} \mathrm{C}-(N)-80^{\circ} \mathrm{C}-(I)$, were synthesized and 
purified at the Institute of Chemistry, Military University of Technology, Warsaw. The purity of the compounds, checked by the chromatography, was better than $99.5 \%$. The samples were placed in the LINKAM cell consisting of two flat indium-tin oxide (ITO) coated glass plates with a spacing of $5 \mu \mathrm{m}$. Due to the rubbed polyimide treatment of the electrode surfaces, the nematic molecules were oriented planar in respect to the electrodes. The probing sinusoidal voltage of the HP 4284A Precision LCR-meter up to $20 V_{\text {rms }}$, at $1 \mathrm{kHz}$, was applied and the cell capacitance as a function of the applied voltage was recorded. The voltage step was $20 \mathrm{mV}$ in the vicinity of the Freedericksz transition and $200 \mathrm{mV}$ for the higher voltage.

\section{Results and discussion}

Figure 2 presents the voltage dependence of the capacitance of the planar cell filled with a pure $8 \mathrm{OCB}$ in the nematic phase. The Freedericksz transition at about $1 \mathrm{~V}$ occurs and on the basis of the voltage threshold value $U_{\text {th }}$ and that of the dielectric anisotropy (taken from our earlier measurements [6]), the value of the splay elastic constant $K_{11}$ at different temperatures can be calculated according to Eq. (1). Figure 3 shows that, as predicted by Eq. (3), the $\left(C-C_{\perp}\right) / C_{\perp}$ dependence on $U^{-1}$ is quite good linear for the voltages much higher than the threshold value. From the slopes of these dependences, the bend elastic constant $K_{33}$ at different temperatures can be calculated with the use of Eq. (4). It should be stressed here that in the temperature range below $66^{\circ} \mathrm{C}$, i.e., in the smectic $A$ phase of $8 \mathrm{OCB}$, the voltage of any value (from the available range of $0-20 \mathrm{~V}$ ) gives no detectable change in the capacity of the sample. Like for other smectic $A$ phases of pure mesogenic compounds, the induction of any deformation in $S_{A}$ phase of $8 \mathrm{OCB}$ by electric field is extremely hard and in the used range of voltage - just impossible.



Fig. 2. Voltage dependence of the capacitance of the planar 8OCB nematic cell at different temperatures (step $\left.1^{\circ} \mathrm{C}\right)$. 


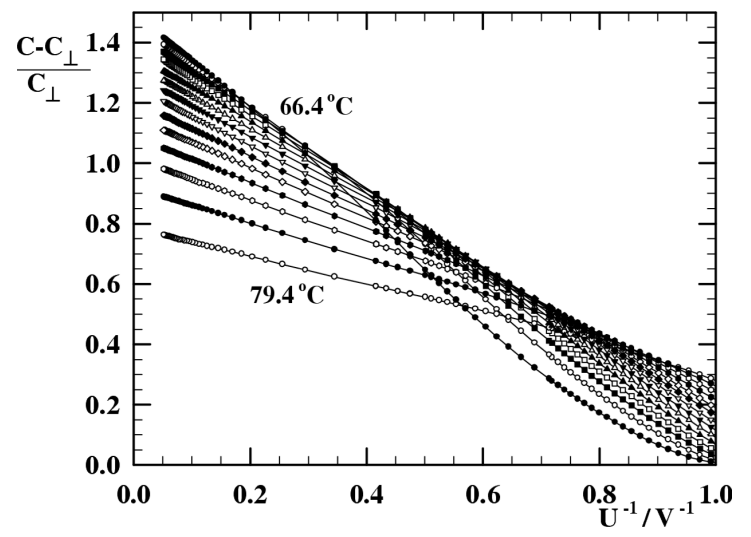

Fig. 3. Dependence of $\left(C-C_{\perp}\right) / C_{\perp}$ on $U^{-1}$ for nematic $8 \mathrm{OCB}$ at different temperatures $\left(\right.$ step $\left.1^{\circ} \mathrm{C}\right)$.



Fig. 4. Temperature dependence of the splay $\left(K_{11}\right)$ and bend $\left(K_{33}\right)$ elastic constants of 6OCB (a) and 8OCB (b). The solid line corresponds to the best fitting of the function $\delta K_{33} \sim\left(T-T^{*}\right)^{-\alpha}$ to the experimental $K_{33}$ values $\left(\alpha=0.98, T^{*}=66^{\circ} \mathrm{C}\right)$.

Figure 4 presents a temperature dependence of the splay and bend elastic constants measured for $6 \mathrm{OCB}$ and $8 \mathrm{OCB}$. The results agree well with the data 
published for the compounds [25]. A critical-like temperature dependence of $K_{33}$, observed for $8 \mathrm{OCB}$ in the vicinity of the nematic to smectic $A$ phase transition, is due to the formation of smectic-like layers in the nematic phase close to the $N-S_{A}$ transition. As the bend deformation of smectic layers must lead to a change of the effective thickness of the layers, the deformation described by the elastic constant $K_{33}$ is in practice impossible to be induced in smectic $A$ phase. So, in such case, a "normal" behavior of $K_{33}$ is an escape to infinity at the temperature of the phase transition. The temperature dependence of $K_{33}$ constant can be then presented as a sum of two components:

$$
K_{33}=K_{33}^{N}+\delta K_{33},
$$

where $K_{33}^{N}$ is the bend elastic constant with the temperature dependence corresponding to the situation of the lack of presmectic effects and $\delta K_{33}$ is the component provided by the existence of short-range order resulting in the smectic-like layers formation in the nematic phase. The dependence of $\delta K_{33}$ component on temperature can be presented in the following form [10]:

$$
\delta K_{33} \sim\left(T-T^{*}\right)^{-\alpha},
$$

where $T^{*}$ is the temperature of the second order $N-S_{A}$ phase transition. The solid line in Fig. 4 corresponds to the best fit of Eq. (6) to the experimental $K_{33}$ data, where the background $K_{33}^{N}(T)$ (similar to $K_{11}(T)$ dependence for $8 \mathrm{OCB}$, see Fig. 4) was taken into account. The obtained value of $T^{*}=66^{\circ} \mathrm{C}$ is equal to the temperature of $N-S_{A}$ phase transition, which is expected for the transition of the second order. The value of the critical exponent $\alpha=0.98$, although appears in the literature, will not be discussed here because of many factors which may influence on the $\alpha$ value, as showed, for example, by Cladis [26].

The elastic constant $K_{11}$ shows no presmectic effects because a splay deformation is, in principle, possible in the layered $S_{A}$ phase [10]. Although possible, the Freedericksz transition, in general, is not observed in the smectic $A$ phase.

Now, let us consider the elastic properties of $8 \mathrm{OCB}+6 \mathrm{OCB}$ mixtures, for which the phase diagram is presented in Fig. 5. For the molecules ratios $Y=$ $N_{6 \mathrm{OCB}} / N_{8 \mathrm{OCB}}$ between about 0.36 and 0.43 , the re-entrant nematic phase $\left(N_{R}\right)$ appears. For $Y<0.36$, the crystallization of the mixtures prevent an appearance of $N_{R}$ phase. Nevertheless, as we will see later, a gradual evolution of the elastic properties of $S_{A}$ phase concerns the whole range of $Y$ value, where the smectic $A$ phase in the mixtures exists. In the smectic $A$ phase composed of the mixture of $8 \mathrm{OCB}$ and $6 \mathrm{OCB}$ molecules, the Freedericksz transition can be induced by applying an electric field of an appropriate strength. As an example, the data obtained for the mixture of concentration $Y=0.300$ are presented in Fig. 6 to illustrate the phenomenon.

In the temperature range of existence of the nematic phase one observes, as expected, the Freedericksz transition at about $1 \mathrm{~V}$ and the voltage threshold value $U_{\text {th }}$ only slightly depends on temperature up to the transition to the smectic 


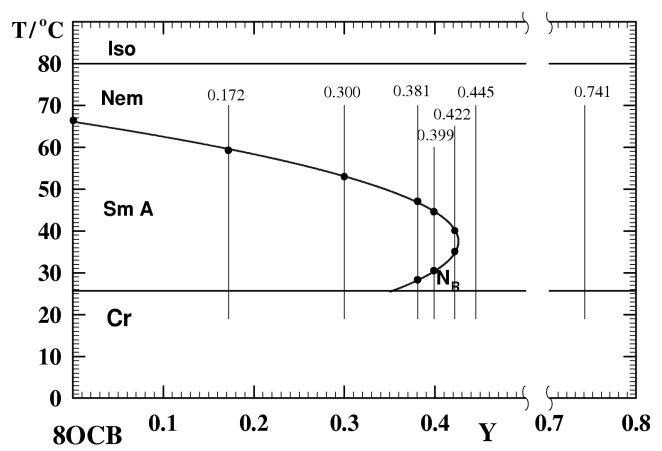

Fig. 5. The phase diagram for $8 \mathrm{OCB} / 6 \mathrm{OCB}$ mixtures. $Y$ denotes the molecules ratio $N_{6 \mathrm{OCB}} / N_{8 \mathrm{OCB}}$. The elastic constants were measured for $Y$ values marked in the diagram.



Fig. 6. Voltage dependence of the capacitance of the planar cell filled with 8OCB/6OCB mixture of $Y=0.300$. The Freedericksz transition appears in the smectic $A$ phase of the mixture.

phase. So, one expects the lack of the presmectic effects in the splay deformation in the nematic phase of the mixture. On the other hand, as $C(U)$ dependence above the voltage threshold value is connected to the elastic constant $K_{33}$, one can see in Fig. 6 that a few degree before the transition to smectic $A$ phase, the pretransitional effects manifest themselves very clearly.

In smectic $A$ phase of the mixture of $Y=0.300$, the Freedericksz transition appears for a much higher voltage than $1 \mathrm{~V}$, and, as it is seen in Fig. 6, the threshold voltage strongly depends on temperature. Because the splay elastic constant is proportional to the square of $U_{\mathrm{th}}$, one may expect here a quite high value of the elastic constant $K_{11}$. Indeed, it is seen in Fig. 7, the splay elastic constant, measured in the smectic $A$ phase of $8 \mathrm{OCB}+6 \mathrm{OCB}$ mixtures, attains the values of about one order of magnitude higher than those measured in the nematic phase of the mixtures. 


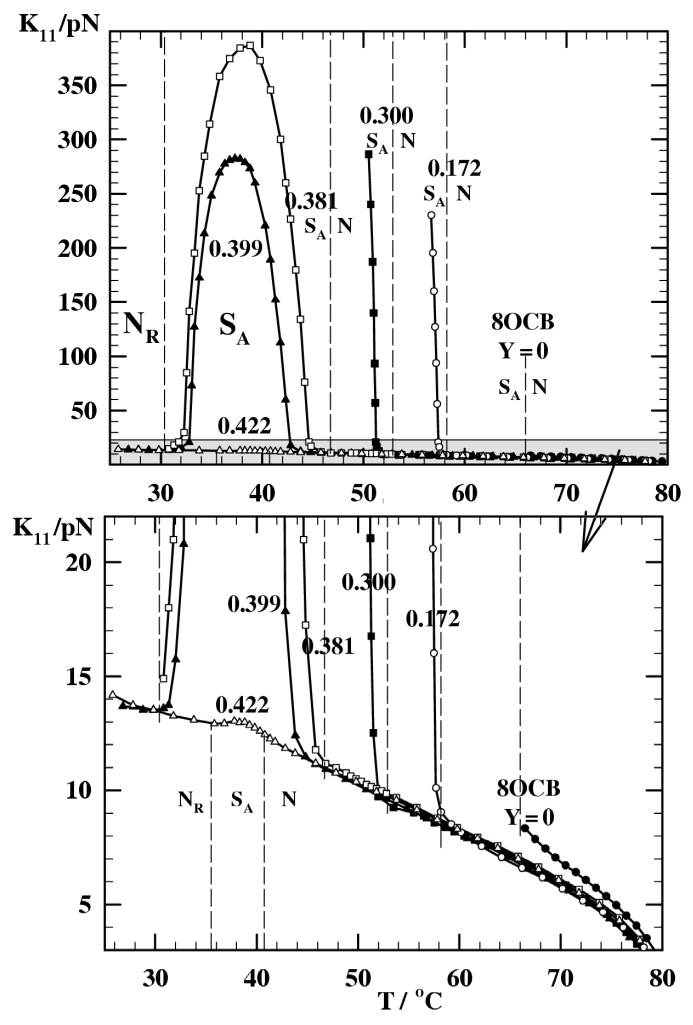

Fig. 7. The splay elastic constant measured in nematic and smectic $A$ phases of $8 \mathrm{OCB} / 6 \mathrm{OCB}$ mixtures as a function of temperature.

The results presented in Fig. 7 confirm our earlier expectations that, similarly to the pure nematic $8 \mathrm{OCB}$, there are no presmectic effects in the temperature dependence of the elastic constant $K_{11}$ measured in $8 \mathrm{OCB}+6 \mathrm{OCB}$ mixtures and that it concerns the whole concentration range of the mixtures studied.

The temperature range, in which the deformations can be induced inside the smectic $A$ phase of the mixtures, strongly depends on the mixture concentration. From one side, there is a total lack of the deformation in the smectic phase of pure $8 \mathrm{OCB}(Y=0)$. From the other side, there are the mixtures $(Y=0.381$, 0.399, and 0.422) situated at the end of the parabola from Fig. 5, in which the deformations in the smectic $A$ phase by external electric field can be performed in the whole temperature range of existence of the phase. Of course, between these two extremities, there are intermediate cases, as it is shown in Fig. 7. So, as a number of $6 \mathrm{OCB}$ molecules increases, we are dealing with an evolution of the elastic properties of the smectic $A$ phase in $8 \mathrm{OCB}+6 \mathrm{OCB}$ mixtures.

Figure 8 presents a temperature dependence of the bend elastic constant measured in $8 \mathrm{OCB}+6 \mathrm{OCB}$ mixtures. For low contents of $6 \mathrm{OCB}$ in the mixtures, 


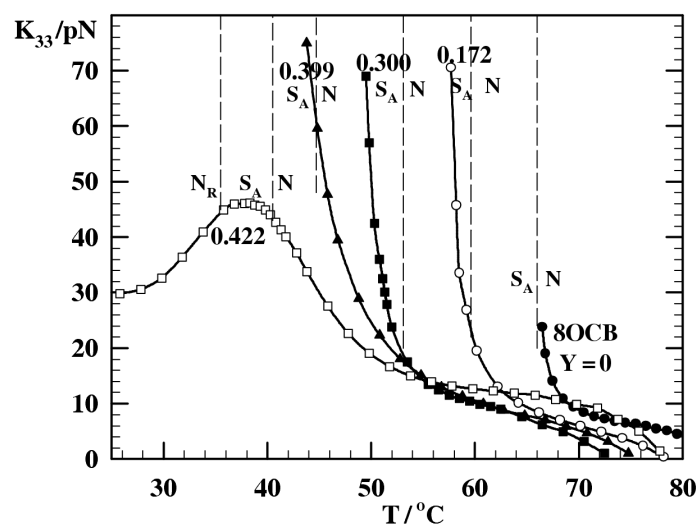

Fig. 8. The bend elastic constant measured in nematic and smectic $A$ phases of $8 \mathrm{OCB} / 6 \mathrm{OCB}$ mixtures as a function of temperature.

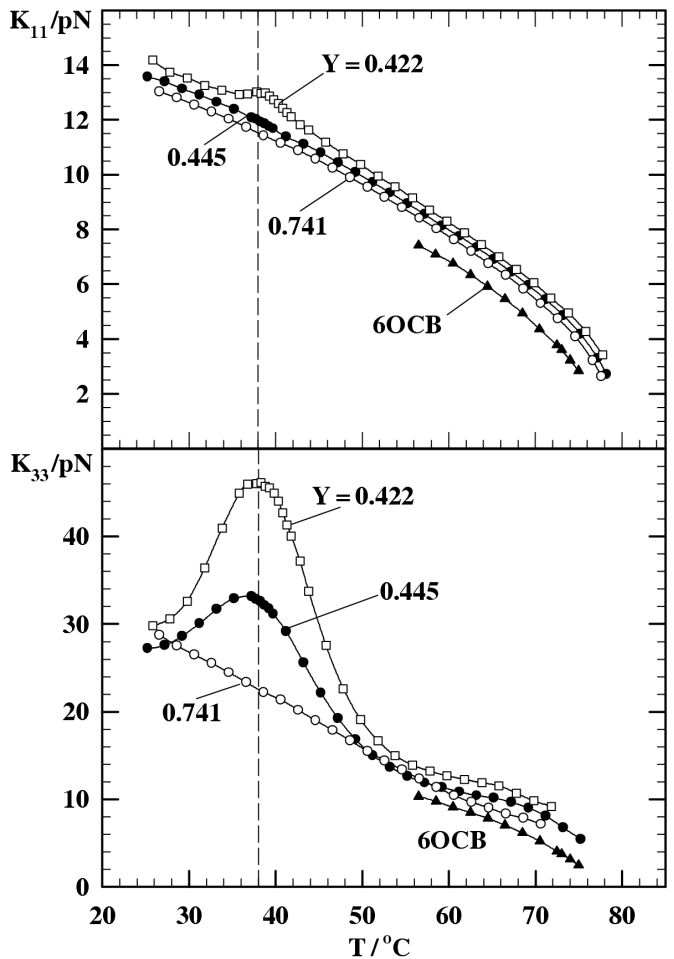

Fig. 9. Temperature dependence of the splay and bend elastic constants measured for $8 \mathrm{OCB} / 6 \mathrm{OCB}$ mixtures beyond the parabola of existence of the smectic $A$ phase. For comparison the dependence for the constants measured for the limit concentration $Y=0.422$ is added. 
the $K_{33}(T)$ dependence shows a critical-like behavior but the asymptote, which for pure $8 \mathrm{OCB}$ is the temperature of $N-S_{A}$ transition, for the mixtures shifts towards an inside of the smectic phase, and $K_{33}(T)$ dependence continuously goes through the $N-S_{A}$ transition. For higher concentrations of $6 \mathrm{OCB}$, the $K_{33}(T)$ dependence loses its critical character, and for $Y=0.422$ it was possible to determine the bend elastic constant in the whole temperature range of smectic $A$ existence.

Figure 9 shows that beyond the parabola of existence of the smectic $A$ phase in $8 \mathrm{OCB}+6 \mathrm{OCB}$ mixtures, i.e., for $Y>0.43$, the influence of the smectic ordering on the nematic elastic properties manifests itself in the bend deformations only. In the $K_{11}$ temperature dependence obtained for $Y=0.445$, there are no traces of influence of the proximity of smectic $A$ phase, in contrary to the $K_{33}(T)$ dependence, which singular behavior ostentatiously transfers to the nematic phase and even for $Y=0.741$ one can notice a shadow of that influence.

\section{Acknowledgment}

The work was partially supported by the Center of Excellence for Magnetic and Molecular Materials for Future Electronics within the European Commission Contract No. G5Ma-CT-2002-04049.

\section{References}

[1] P.E. Cladis, Phys. Rev. Lett. 35, 48 (1975).

[2] P.E. Cladis, in: Handbook of Liquid Crystals, Eds. D. Demus, J. Goodby, G.W. Gray, H.-W. Spiess, V. Vill, Vol. 1, Wiley - VCH, Weinheim 1998, p. 391.

[3] A. Kubono, K. Yoshino, T. Ninomiya, R. Akiyama, K. Tanaka, Liq. Cryst. 29, 1089 (2002).

[4] J. Jadżyn, G. Czechowski, Phys. Rev. E 64, 052702 (2001).

[5] S. Urban, R. Dąbrowski, B. Gestblom, A. Kocot, Liq. Cryst. 27, 1675 (2000).

[6] J. Jadżyn, G. Czechowski, Liq. Cryst. 4, 157 (1989).

[7] R. Nozaki, T.K. Bose, S. Yagihara, Phys. Rev. A 46, 7733 (1992).

[8] A. Żywociński, J. Phys. Chem. B 103, 3087 (1999).

[9] A.P. Filippov, J. Lindau, Polymer Sci. A 42, 743 (2000).

[10] P.G. de Gennes, J. Prost, The Physics of Liquid Crystals, Clarendon, Oxford 1993.

[11] P.E. Cladis, R.K. Bogardus, D. Aadsen, Phys. Rev. A 78, 2292 (1978).

[12] A.R. Kortan, H.V. Kanel, R.J. Birgeneau, J.D. Lister, Phys. Rev. Lett. 47, 1206 (1981)

[13] D. Guillon, A. Skoulios, J. Phys. (Paris) 45, 607 (1984).

[14] L. Longa, W.H. de Jeu, Phys. Rev. A 28, 2380 (1983).

[15] N.V. Madhusudana, J. Rajan, Liq. Cryst. 7, 31 (1983).

[16] A.S. Govind, N.V. Madhusudana, Liq. Cryst. 27, 215 (2000). 
[17] P.E. Cladis, P.L. Finn, J.W. Goodby, in: Liquid Crystals and Ordered Fluids, Eds. A.C. Griffin, J.F. Jahnson, Vol. 4, Plenum, New York 1984, p. 203.

[18] S.A. Ivanov, V.V. Belaiev, R.A. Zeinalov, B.I. Ostrovskij, Zh. Exp. Theor. Fiz. 91, 560 (1986).

[19] S. Bhattacharya, S.V. Letcher, Phys. Rev. Lett. 44, 414 (1980).

[20] A.S. Sailaja, K. Venu, V.S.S. Sastry, Mol. Cryst. Liq. Cryst. 250, 177 (1993).

[21] H. Gruler, T.J. Scheffer, G. Meier, Z. Naturforsch. A 27, 966 (1972).

[22] T. Uchida, Y. Takahashi, Mol. Cryst. Liq. Cryst. 72, 133 (1981).

[23] P. Chattopadhyay, S.K. Roy, Mol. Cryst. Liq. Cryst. 257, 89 (1994).

[24] Y. Zhou, S. Sato, Jpn. J. Appl. Phys. 37, 4397 (1997).

[25] M.J. Bradshaw, E.P. Raynes, J.D. Bunning, T.E. Faber, J. Phys. (Paris) 46, 1513 (1985).

[26] P.E. Cladis, Phys. Rev. Lett. 31, 1200 (1973). 